Revue internationale P.M.E.

Économie et gestion de la petite et moyenne entreprise

\title{
Des stratégies aux comportements spatiaux de l'entreprise : l'insertion locale des PME
}

\section{Nathalie Bertrand}

Volume 12, numéro 1-2, 1999

URI : https://id.erudit.org/iderudit/1008651ar

DOI : https://doi.org/10.7202/1008651ar

Aller au sommaire du numéro

Éditeur(s)

Presses de l'Université du Québec

ISSN

0776-5436 (imprimé)

1918-9699 (numérique)

Découvrir la revue

Citer cet article

Bertrand, N. (1999). Des stratégies aux comportements spatiaux de l'entreprise : l'insertion locale des PME. Revue internationale P.M.E., 12(1-2), 85-106. https://doi.org/10.7202/1008651ar
Résumé de l'article

De nombreuses contributions ont été réalisées en économie industrielle sur le rapport de l'entreprise à l'espace et aux territoires. Cet article, par une approche microéconomique, s'interroge sur l'influence de la stratégie sur le comportement spatial de l'entreprise. L'analyse s'articule autour de trois axes, l'entrepreneur, la nature des relations de l'entreprise et leur localisation.

Trois comportements spatiaux sont ainsi identifiés, distinguant successivement des comportements localisés, impliquant la proximité spatiale de l'entreprise; des comportements délocalisés pour lesquels les relations locales sont ténues enfin, des comportements territorialisés pour lesquels l'entreprise est insérée dans des formes de coopérations territorialisées. 


\title{
Des stratégies aux comportements spatiaux de l'entreprise: l'insertion locale des PME
}

Nathalie BERTRAND

Cemagref-Développement des territoires montagnards, Grenoble

\section{MOTS CLÉS}

\section{Entreprise - Entrepreneur - Stratégie \\ Comportement spatial - Coordination}

\begin{abstract}
RÉSUMÉ
De nombreuses contributions ont été réalisées en économie industrielle sur le rapport de l'entreprise à l'espace et aux territoires. Cet article, par une approche microéconomique, s'interroge sur l'influence de la stratégie sur le comportement spatial de l'entreprise. L'analyse s'articule autour de trois axes, l'entrepreneur, la nature des relations de l'entreprise et leur localisation.

Trois comportements spatiaux sont ainsi identifiés, distinguant successivement des comportements localisés, impliquant la proximité spatiale de l'entreprise; des comportements délocalisés pour lesquels les relations locales sont ténues; enfin, des comportements territorialisés pour lesquels l'entreprise est insérée dans des formes de coopérations territorialisées.
\end{abstract}

\section{ABSTRACT}

Many works have been done on enterprises spatial relationships, especially by industrial economists. This article, with an micro-economic approach, focuses on strategy influence on enterprise spatial behaviour. The analysis uses three axes, enterpreneur, enterprise relations nature, enterprise relations location.

\section{L'AUTEURE}

Nathalie Bertrand est ingénieure du GREF (génie rural, des eaux et forêts) et détient un doctorat en économie de l'Université Pierre-Mendès-France (Grenoble). Elle travaille au Cemagref dans l'unité DTM, Développement des territoires montagnards. Ses principaux domaines de recherche portent sur les stratégies d'entreprises, les réseaux d'acteurs et les dynamiques territoriales. Adresse : 2, rue de la Papeterie, BP 76, Domaine universitaire, 38402 St-Martin d'Hères, Cedex, France. Téléphone: 3304767627 42. Télécopieur: 330476513803. Courriel : nathalie.bertrand@grenoble.cemagref.fr 
Three spatial behaviours have been identified: localized behaviours, involving enterprise spatial proximity; delocalized behaviours for which local relationships are slight and embedded behaviours, where enterprises are involved in territorial cooperation.

\section{RESUMEN}

La relación de la empresa con el espacio y el territorio ha sido objeto de numerosas contribuciones en economía industrial. En este artículo, a partir de una perspectiva microeconómica, el autor plantea la influencia que puede tener la estrategia sobre la conducta espacial de una empresa. Se analiza el problema teniendo en cuenta el empresario, la naturaleza de las relaciones de la empresa y la ubicación de ésta.

Se han identificado tres tipos de conducta espacial : los comportamientos localizados, que implican la proximidad espacial de la empresa ; los que implican un alejamiento pero que incluyen las relaciones locales y finalmente los comportamientos territorializados, en los que la empresa se inserta en formas de cooperación territorializadas.

\section{ZUSAMMENFASSUNG}

In der Industriewirtschaft wurden viele Beiträge geliefert im Zusammenhang mit der Unternehmung und ihrem räumlichen Verhalten. Dieser Artikel durchleuchtet den Einfluss der Strategie auf das räumliche Verhalten der Unternehmung anhand des mikroökonomischen Ansatzes.

Die Analyse gliedert sich in 3 Achsen: Unternehmer, Beziehungsnetz der Unternehmung und ihre Lokalisierung.

Es werden 3 räumliche Verhaltensweisen festgestellt, die sich wie folgt unterscheiden: lokalisierte Verhaltensweisen, die stark mit der unmittelbar räumlichen Nähe der Unternehmung verbunden sind; delokalisierte Verhaltensweisen, für die ein lokales Beziehungsnetz gehalten wird; territoriale Verhaltensweisen, für welche die Unternehmung in territoriale Kooperationsformen eingegliedert wird.

La question de l'organisation spatiale des entreprises est au centre des interrogations actuelles d'économie industrielle. L'espace n'apparaît plus comme un simple cadre dans lequel s'inscrivent des phénomènes économiques, mais comme un facteur parmi d'autres de leurs modes d'organisation et de leur dynamisme (Rallet et Torre, 1995). L'abondance des publications interpelle l'aménagement du territoire sur les localisations d'activités industrielles, l'insertion locale des entreprises, mais aussi la structuration du système productif.

De nombreuses contributions ont été réalisées en économie industrielle, en termes d'externalités (Lecoq, 1992), de diffusion de l'innovation (Maillat, 1992), ou encore de systèmes productifs locaux (Courlet et Pecqueur, 1991), insistant sur 
la mutuelle influence des entreprises et des constructions sociales et institutionnelles que constituent les «territoires ». Or, selon la stratégie ${ }^{1}$ affichée par l'entreprise, les choix en matière d'activité et d'organisation sont différents, tant par la nature des relations tissées avec l'environnement que par leurs implications spatiales. Quelle est alors la construction de l'espace des relations de l'entreprise ? Quelle est son insertion locale? La question de l'influence de la stratégie sur le comportement spatial de l'entreprise est ouverte. Elle constitue l'objet de cet article.

Des éléments de réponse sont trouvés dans la recherche que nous avons menée sur l'entreprise industrielle - essentiellement sur des petites entreprises et des toutes petites entreprises ${ }^{2}-$ et son insertion locale ${ }^{3}$. Centrée sur la question rurale, elle a pu éclairer cette question grâce à l'analyse de ses flux. Nous tentons ici d'en restituer les principaux résultats en précisant les postulats qui nous ont permis d'y aboutir et la façon dont nous avons approché la notion de stratégie d'entreprise. Notre analyse des comportements spatiaux s'est articulée autour de trois axes : l'entrepreneur, la nature des coordinations de l'entreprise, leurs localisations. Enfin, les flux d'information ont été privilégiés. Ils sont, en effet, d'autant plus importants que l'entreprise est de petite taille et que l'accès lui en est difficile.

Trois comportements spatiaux ont été ainsi identifiés au regard des «préférences » de l'entrepreneur et de la nature des relations de l'entreprise aux acteurs (ou institutions) de l'environnement. Nous n'en mentionnons que les grandes tendances, sans préciser ici les parcours possibles au cours de l'évolution de l'entreprise. Cela a le mérite de la clarté et constitue un passage obligé pour une meilleure compréhension du phénomène.

Nous distinguons donc successivement des comportements localisés, impliquant la proximité spatiale de l'entreprise ; des comportements délocalisés pour lesquels l'espace des relations de l'entreprise est discontinu, et les relations locales, ténues et fonctionnelles; enfin, des comportements territorialisés où l'espace des relations peut également être discontinu, mais pour lesquels l'entreprise est insérée dans des formes de coopérations (ou réseaux) territorialisées.

1. Nous retiendrons comme définition de la stratégie celle donnée par Porter (1986, p. 11): «La stratégie consiste à rechercher une position favorable dans un secteur, scène où la concurrence se manifeste principalement. La stratégie vise à l'obtention d'une position rentable et durable au milieu des forces qui définissent le cadre concurrentiel du secteur.»

2. Que nous nommerons dans le reste du texte respectivement, PE et TPE.

3. N. Bertrand (1996), «Ancrage spatial des entreprises en milieu rural : de l'espace fonctionnel à l'espace territoire », Thèse de doctorat en économie appliquée, 12 juillet, Université Pierre-Mendès-France Grenoble II, 320 p. 


\section{Les préférences de l'entrepreneur et les relations à l'environnement}

\section{Les postulats de la recherche}

La notion d'entreprise « industrielle » est le plus souvent identifiée statistiquement aux établissements de plus de 10 salariés, des $\mathrm{NAF}^{4} 10$ à 45 . Les entreprises de taille inférieure sont considérées comme artisanales. Or, la frontière entre ces deux ensembles est floue (Jaeger, 1982) : la tertiarisation de l'industrie rend plus ténue la limite entre secteur industriel et secteur tertiaire ; l'hétérogénéité de leurs caractéristiques remet en cause sa pertinence. Le seul critère de taille apparaît donc insuffisant pour déclarer une entreprise artisanale ou non. Nous avons alors retenu comme déterminant le comportement stratégique de l'entreprise, en élargissant la notion d'entreprise industrielle aux entreprises de moins de neuf salariés, et aux entreprises du tertiaire «supérieur », services aux entreprises, services informatiques par exemple ${ }^{5}$.

Notre recherche s'est, de plus, intéressée à la place de l'entrepreneur. Dans l'histoire de la pensée économique, après avoir été ignoré, il s'impose rapidement comme un acteur déterminant. Il est un catalyseur au centre de la dynamique économique (Say, 1803); il organise les facteurs de production ; il est à l'origine des « combinaisons nouvelles» (Knight, 1921 ; Schumpeter, 1935), d'innovations, de nouveaux marchés et formes d'organisation. Les caractéristiques socioéconomiques du chef d'entreprise témoignent de l'hétérogénéité du monde patronal des PE et des TPE, et traduisent différents modes de gestion. Bauer (1988) qualifie les entrepreneurs par la nature de leurs projets, les valeurs qu'ils développent et le mode de fonctionnement qu'ils donnent à leur entreprise. Selon leurs origines sociales, leur capital culturel et relationnel (autodidacte, diplômé d'université, d'une grande école, membre d'un corps d'État), ou leur identité patronale, les dirigeants jouent divers rôles (Weber, 1988).

L'importance du chef d'entreprise et son influence sur la structure nous autorisent dans la démonstration à identifier l'entreprise à l'entrepreneur, d'autant

4. Nomenclature d'activités françaises 10 à 45 (au $1^{\text {er }}$ janvier 1993): industries agroalimentaires, de métaux, de l'énergie, de la chimie, caoutchouc et matières plastiques, verres et matériaux de construction, papier et carton, mécanique, électricité et électronique, construction automobile, textile, cuir et chaussures, bois et ameublement, industries diverses, bâtiments-génie civil-agriculture, pharmacie et presse-édition.

5. D'après les travaux de Jaeger (1982) sur l'artisanat et les pratiques de leurs responsables, nous considérons industrielles les « entreprises intermédiaires » et les «petites entreprises capitalistes ». Les premières sont généralement en croissance et présentent un processus de production qui s'appuie sur une croissance légère du capital. Les secondes affichent une division et une organisation du travail, une obligation de rendement du capital et un début de production en série. 
plus facilement que la gestion des petites entreprises est fortement personnalisée (Julien et Marchesnay, 1987). Nous traduirons donc la stratégie menée par les buts du dirigeant, ses choix en matière de position concurrentielle et de coordination à l'environnement.

Le dernier postulat porte sur la représentation de l'espace dans notre interprétation des comportements spatiaux. Il rejette son assimilation à la seule distance qui ne peut refléter les relations complexes de l'homme (et des institutions) à l'espace. Le concept de territoire nous permet ainsi de rendre compte des construits sociaux, économiques et culturels avec lesquels l'entreprise interfère. Il est cependant diversement défini. Les géographes l'identifient à un «corps à corps » entre la nature, les hommes et leurs rapports sociaux (Raffestin, 1986). Les économistes l'associent à une interpénétration industrie-société locale dans les districts (Becattini, 1989 ; Bagnasco, 1977 ; Garofoli, 1992) et les systèmes productifs locaux (Courlet, 1990 ; Courlet et Pecqueur, 1991). Ils l'approchent également par les milieux innovateurs dans les travaux du GREMI (Perrin, 1992). Nous nous y intéresserons à travers l'implantation de l'entreprise et les relations qu'elle y tisse, comme ensemble de proximités sociales, culturelles, économiques, organisationnelles et géographiques que l'entreprise met en œuvre.

\section{Buts de l'entrepreneur, position concurrentielle et choix de coordinations}

De nombreuses analyses existent sur les prises de décision de l'entrepreneur et ses logiques d'action. Cependant, deux idées-forces, empruntées aux sciences de gestion et à l'économie industrielle nous permettent d'approcher les stratégies des entreprises au regard de notre questionnement : les préférences de l'entrepreneur (Marchesnay, 1986 ; 1991) et sa référence à l'environnement (Lawrence et Lorsch, 1973 ; Martinet, 1983 ; Porter, 1986).

S'il y a recherche de profit, sa maximisation n'est pas la seule fin de l'entrepreneur. Il poursuit d'autres objectifs et présente un ensemble de buts hiérarchisés. Ses préférences orientent la conduite de l'entreprise, son positionnement sur un secteur d'activité, ses relations à l'environnement. Pour les PE et les TPE que nous avons observées, nous distinguons, à la suite de Julien et Marchesnay (1987), trois autres buts qui, articulés, conditionnent l'efficience recherchée par l'entrepreneur : 1) la pérennité de l'entreprise : l'entrepreneur recherche la survie de l'entreprise à son créateur, la transmission à des héritiers ; 2) l'indépendance : l'entrepreneur est mû par le «désir d'être son propre patron ». Ce souhait d'indépendance se manifeste dans les logiques financières, industrielles (partenariat, diversification, développement) ou même institutionnelles (relation avec la profession, les collectivités locales). Il conduit, dans certains cas, à un refus de croissance ; 3) la puissance ou le pouvoir qui se traduisent par le désir d'accomplissement de l'entrepreneur 
et par sa volonté d'assurer la croissance de l'entreprise : extension du domaine d'action avec une efficacité croissante, valorisation des capitaux accumulés et engagés. La vision de l'environnement est alors opportuniste.

Au-delà des choix de l'entrepreneur, la stratégie se traduit également par la position concurrentielle recherchée. Le dirigeant peut jouer sur des coûts faibles ou sur une différenciation (Porter, 1986). Dans le premier cas, l'entreprise tend à produire à faible coût. C'est la recherche d'économies d'échelle, de technologies exclusives ou d'un accès préférentiel aux matières premières. Le créneau peut être lié à des compétences spéciales. Il n'est pas limité à un domaine de fabrication et met en œuvre des compétences développées très tôt par des savoir-faire pointus. Les petites entreprises rencontrées jouent peu sur ces seuls critères.

Dans le second cas, l'entreprise différencie sa production sur une demande client par la sophistication du produit, par une marque ou une image, ou encore par un accès limité au marché. La différenciation n'existe que parce que le consommateur la reconnait ; elle est rémunérée par un accroissement de la valeur de l'offre. L'entreprise se place alors sur un créneau (ou niche « écologique ${ }^{6} »$ ). Celui-ci peut également être constitué par un marché spécifique détenu par l'entreprise. L'entreprise s'appuie sur une connaissance approfondie du marché. Elle connaît bien les demandeurs; elle est parfaitement connue de la clientèle potentielle. Le marché n'est pas assez grand ni suffisamment attrayant pour tenter les concurrents (bien que rien ne puisse définitivement être considéré comme une "chasse gardée »). Le plus grand danger réside dans le fait de devenir un marché de masse. De nombreuses petites entreprises se situent sur des créneaux intermédiaires, alliant marché spécialisé et compétences pointues.

La stratégie de l'entrepreneur se traduit également par des formes de coordination qui échappent à la relation de « pur » marché ou à la coordination hiérarchique (Richardson, 1972). Celles-ci font référence à des mondes d'action implicites qui permettent aux acteurs de travailler, d'échanger ou de produire. Salais et Torper (1993) proposent quatre types de coordination à partir des travaux de Boltanski et Thévenot (1991) selon la nature des biens échangés et les compétences qui sont nécessaires et que chaque acteur suppose chez l'autre. La première est industrielle. Elle est fondée sur l'efficacité productive, la performance et la capacité des acteurs à répondre à la demande. La concurrence entre les producteurs s'effectue par le prix, seul argument de différenciation que retient le demandeur. La deuxième est marchande. Elle est caractérisée par des rapports de concurrence et par son caractère atemporel. La troisième est qualifiée de personnelle. Elle se construit sur des relations personnalisées entre acteurs économiques qui disposent d'une identité connue de part et d'autre. Les rapports entre acteurs (demandeurs ou producteurs)

6. Ce parallèle biologique est proposé par Drucker (1985) comme un « milieu offrant à une population bien déterminée des conditions relativement stables». 
reposent sur une entente mutuelle et des relations de confiance ${ }^{7}$ entre les acteurs impliqués, leurs relations de considération et sur un langage commun. Enfin, la dernière coordination est qualifiée d'immatérielle et engage un monde de création, de nouvelles technologies et familles de produits. Certaines de ces coordinations sont de nature coopérative (ou de nature résiliaire ${ }^{8}$ ), impliquant des formes d'interdépendance entre les acteurs.

\section{La démarche}

La recherche s'est appuyée sur un travail de terrain mené auprès d'entrepreneurs de 62 entreprises des Alpes-de-Haute-Provence, des Hautes-Alpes et de Lozère. La sélection des entreprises a été effectuée à partir des fichiers de la Chambre de commerce et d'industrie et ceux de la Chambre des métiers. Elle a été complétée par les avis de chargés de mission des Chambres ou d'organismes de développement contactés, établis à partir de quatre paramètres : la dynamique de l'entreprise (viabilité dans le milieu), l'envergure locale, nationale, internationale, etc., de ses marchés, la personnalité de l'entrepreneur, sa démarche innovante (technique, produit, commerciale). Les entreprises retenues ont alors fait l'objet d'un dernier tri selon les secteurs présents, leur répartition géographique, la localisation du centre de décision, leur taille et leur âge et l'origine des créateurs. Chaque entretien a fourni une part de l'information nécessaire à l'élaboration de « profils types » d'entreprises. Les tableaux 1 et 2 permettent de situer leur proportion relative dans l'échantillon choisi, selon les tailles d'entreprises (tableau 1) et les secteurs industriels où l'agroalimentaire se démarque comme un des secteurs traditionnels du milieu rural (tableau 2).

Les entretiens semi-dirigés ont abordé trois thèmes : le profil de l'entreprise (son historique, son organisation et les motivations à l'installation de l'entrepreneur), la stratégie développée et la traduction spatiale des coordinations. L'analyse de la stratégie des PE et des TPE étudiées s'est appuyée sur les buts de l'entrepreneur, le positionnement de l'entreprise par rapport à la concurrence et les coordinations mises en place. Les stratégies possibles sont différentes ; les comportements spatiaux le seront aussi.

Enfin, une grille de lecture a permis d'analyser le comportement spatial de l'entreprise à partir des pratiques stratégiques de l'entrepreneur, de la nature des

7. Cette confiance est définie par Arrow (1974) comme une institution invisible au même titre que les règles de droit ou les principes éthiques.

8. La notion de réseau adoptée ici est proche de l'une des deux conceptions mises en avant par Joly et Mangematin (1995) : le réseau constitue une forme de coordination caractérisée par une forte interdépendance et par un partage de pouvoir entre les acteurs qui, dans un environnement turbulent, développent des relations stables permettant aux organisations de maîtriser l'accès aux ressources stratégiques. 
TABLEAU 1

Stratégies d'entreprises par taille d'entreprises

\begin{tabular}{lcccc}
\hline $\begin{array}{l}\text { Taille } \\
\text { d'entreprises }\end{array}$ & $\begin{array}{c}\text { Stratégies } \\
\text { artisanales }\end{array}$ & $\begin{array}{c}\text { Stratégies } \\
\text { adaptatives }\end{array}$ & $\begin{array}{c}\text { Stratégies } \\
\text { offensives }\end{array}$ & $\begin{array}{c}\text { Stratégies } \\
\text { commandées }\end{array}$ \\
\hline 0-9 salariés & 6 & 13 & 1 & 2 \\
10-19 salariés & 1 & 10 & 5 & 2 \\
20-49 salariés & & 2 & 10 & 3 \\
50-99 salariés & & & 1 & 1 \\
>à 100 salariés & & & 3 & 2 \\
\hline Total des effectifs & $\mathbf{7}$ & $\mathbf{2 5}$ & $\mathbf{2 0}$ & $\mathbf{1 0}$ \\
\hline
\end{tabular}

\section{TABLEAU 2}

Stratégies d'entreprises selon les secteurs industriels

\begin{tabular}{|c|c|c|c|c|c|}
\hline $\begin{array}{l}\text { Stragégies / } \\
\text { Secteurs industriels }\end{array}$ & $\begin{array}{l}\text { Stratégies } \\
\text { artisanales }\end{array}$ & $\begin{array}{l}\text { Stratégies } \\
\text { adaptatives }\end{array}$ & $\begin{array}{l}\text { Stratégies } \\
\text { offensives }\end{array}$ & $\begin{array}{c}\text { Stratégies } \\
\text { commandées }\end{array}$ & $\begin{array}{c}\text { Total } \\
\text { par secteur }\end{array}$ \\
\hline Agroalimentaire & 3 & 6 & 4 & 1 & 14 \\
\hline Bois et papier & 1 & 3 & 2 & & 6 \\
\hline Presse, édition & 1 & 5 & 1 & & 7 \\
\hline $\begin{array}{l}\text { Caoutchouc } \\
\text { et matières plastiques }\end{array}$ & & & 1 & 1 & 2 \\
\hline Chimie & & 4 & & 3 & 7 \\
\hline Textile et cuir & 1 & 3 & 1 & & 5 \\
\hline \multicolumn{6}{|l|}{ Verre et matériaux } \\
\hline Métaux & 1 & 1 & 2 & 1 & 5 \\
\hline $\begin{array}{l}\text { Économie énergétique, } \\
\text { extraction }\end{array}$ & & & 1 & 1 & 2 \\
\hline \multicolumn{5}{|c|}{ Mécanique, électricité, } & 11 \\
\hline Activité d'études, de & nseil & 1 & & & 1 \\
\hline
\end{tabular}

coordinations, de leurs fondements (ce sur quoi elles sont établies) ainsi que de leur localisation. Les trois paragraphes suivants adoptent ce développement (le tableau 3 en résume les principaux points). Ils précisent l'espace des relations et identifient le mode d'insertion locale de l'entreprise.

\section{Des comportements localisés: les stratégies artisanales}

Les entreprises concernées dépassent rarement huit à neuf salariés et sont créées avec peu de moyens. Elles se développent sur le long terme, parfois sur plusieurs générations et se transmettent : c'est un patrimoine familial. Les investissements sont limités et réalisés au coup par coup; le taux d'endettement de l'entreprise est 
TABleau 3

Des stratégies aux comportements spatiaux de l'entreprise

\begin{tabular}{|c|c|c|c|}
\hline $\begin{array}{l}\text { Stratégie de } \\
\text { l'entreprise }\end{array}$ & $\begin{array}{l}\text { Buts de } \\
\text { l'entrepreneur }\end{array}$ & $\begin{array}{l}\text { Caractéristiques } \\
\text { de l'entreprise }\end{array}$ & $\begin{array}{l}\text { Coordinations } \\
\text { dominantes } \\
\text { et comportement } \\
\text { spatial }\end{array}$ \\
\hline - artisanale & $\begin{array}{l}\text { - pérennité et } \\
\text { indépendance } \\
\text { - entrepreneur } \\
\text { local }\end{array}$ & $\begin{array}{l}-<10 \text { salariés } \\
- \text { familiale } \\
- \text { investissements } \\
\quad \text { limités } \\
- \text { produits sur } \\
\text { mesure }\end{array}$ & $\begin{array}{l}\text { - coordinations } \\
\text { personnelles } \\
\text { - activité limitée à la } \\
\text { proximité spatiale } \\
\text { - comportement } \\
\text { localisé }\end{array}$ \\
\hline - adaptative & $\begin{array}{l}\text { - indépendance } \\
\text { - pérennisation }\end{array}$ & $\begin{array}{l}-<20 \text { salariés } \\
\text { - patrimoniale } \\
\text { - différenciation } \\
\text { - investissements } \\
\text { réguliers } \\
\text { - petites séries } \\
\text { - AOC, label, } \\
\text { image }\end{array}$ & 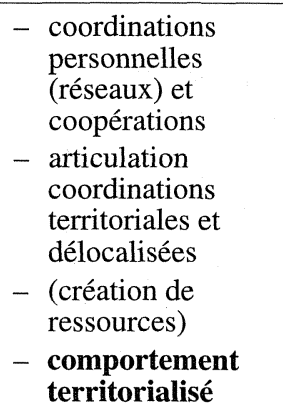 \\
\hline - offensive & $\begin{array}{l}\text { - croissance } \\
\text { (indépendance) }\end{array}$ & $\begin{array}{l}\text { - }>20 \text { salariés } \\
\text { - ouverture } \\
\text { du capital } \\
\text { - investissements } \\
\text { importants } \\
\text { - circuit } \\
\text { commercial } \\
\text { - différenciation }\end{array}$ & $\begin{array}{l}\text { - coordinations } \\
\text { marchandes et } \\
\text { industrielles, } \\
\text { spatialement } \\
\text { distantes } \\
\text { - comportement } \\
\text { délocalisé }\end{array}$ \\
\hline - commandée & - croissance & $\begin{array}{l}\text { - taille variable } \\
\text { - établissement } \\
\text { - circuit } \\
\text { commercial } \\
\text { du groupe } \\
\text { - produit du groupe } \\
\text { et différenciation }\end{array}$ & $\begin{array}{l}\text { - coordinations } \\
\text { marchandes et } \\
\text { industrielles } \\
\text { - espace du groupe } \\
\text { - comportement } \\
\text { délocalisé }\end{array}$ \\
\hline
\end{tabular}

faible; les capitaux sont familiaux. L'entrepreneur, le plus souvent issu du milieu local, est un «artisan » : il maîtrise un ou plusieurs savoir-faire. La «gestion » de personnel, éloignée de ses compétences techniques, lui répugne; l'embauche de nouveaux salariés apparaît comme une étape contraignante qu'il n'est pas enclin à franchir. L'activité est restreinte au potentiel humain dont dispose l'entreprise. La main-d'œuvre est avant tout familiale. Ces entreprises sont en minorité dans l'échantillon (tableau 1), plus présentes en agroalimentaire ou dans le secteur de la presse-édition. 
L'entrepreneur est issu d'une famille d'ébénistes. Il crée en 1980 une entreprise individuelle de deux salariés pour développer un travail d'ébénisterie contemporaine. L'entrepreneur se considère comme un « artisan »: il « vend du savoir-faire »; il crée un mobilier de conception esthétique originale. Les produits sont adaptés à la clientèle, alliant la diversité de création à une souplesse d'adaptation à la demande.

L'évolution de l'entreprise a suivi celle du marché. Au départ, la clientèle est essentiellement privée et locale, voire régionale. Actuellement, avec la baisse du pouvoir d'achat, la clientèle privée ne représente plus que $30 \%$ du chiffre d'affaires, contre $70 \%$ pour les collectivités, les groupements ou les administrations. L'entreprise n'a jamais eu (au dire de l'entrepreneur) une si bonne rentabilité qu'aujourd' hui. Elle se démarque par son métier et par la qualité de ses réalisations.

La façon dont l'entrepreneur crée autour de lui un « tissu » d'entreprises du bois fait cependant sa différence sur le marché. Il répond très peu aux appels d'offre qu'il considère comme insuffisamment pointus pour valoriser son savoir-faire (d'ailleurs, il dit ne pas avoir de concurrence sur les marchés régionaux, mais sur les marchés publics venant de l'extérieur). En moyenne, les deux tiers de son activité résultent de marchés de gré à gré avec des architectes (quand l'architecte veut mettre sa griffe). Un tiers du chiffre d'affaires provient de cotraitances. Celles-ci sont réalisées grâce aux liens privilégiés que tisse l'entreprise avec des entreprises locales du secteur du bois (trois à quatre contrats par an) : complémentarité de compétences sur des contrats communs - qui leur permet de conserver des marchés qu'elles ne pourraient traiter seules -, prêts de matériel, échanges d'information technique et d'information sur les marchés. Ces échanges internes ne sont pas structurés. Ils sont basés sur des relations de confiance (on se connaît bien ; on est tous collègues). Les relations par connaissance (réseaux) interviennent largement dans l'obtention de marchés (réputation de sérieux, de bouche à oreille).

Enfin, l'entreprise, depuis sa création, fait partie de la coopérative «artisanat du bois » du département. Ce mode de commercialisation ne représente plus qu'une faible part de son chiffre d'affaires.

L'entrepreneur a, face à l'incertitude du futur, un désir de pérennité et d'indépendance. Il privilégie la maîtrise technique à la croissance. L'entrepreneur est reconnu pour ses compétences. Les activités de l'entreprise se développent grâce à une diffusion des marchés (et de l'information) par le «bouche à oreille ». Le souci de valorisation de la production conduit l'entreprise vers des productions spécialisées et dédiées à une demande préexistante. Le produit est fait «sur mesure ». Les marchés sont spécifiques et demandent avant tout une maîtrise technique, inventive. Ces entreprises sont artisanales, bien que relevant pour certaines de la Chambre de commerce et d'industrie et non de la Chambre des métiers.

Ces pratiques artisanales s'appuient essentiellement sur des coordinations personnelles, même si certains flux amont font intervenir des coordinations marchandes, comme les approvisionnements. L'activité se limite à une proximité 
spatiale (le plus souvent, le département) et est tournée vers des demandes locales, de natures variées, toujours liées au métier. Il n'y a pas de démarche commerciale particulière : les produits propres, lorsqu'ils existent, se heurtent donc à des difficultés de commercialisation. Faute de débouchés, ils demeurent à l'état de prototypes. Les relations avec la clientèle se fondent sur la réputation de l'entrepreneur et sur son appartenance au territoire. Il s'instaure un langage commun entre le client et l'artisan : ils se connaissent, adaptent la production à la demande et la demande, au savoir-faire de l'artisan. Des coopérations avec d'autres entrepreneurs locaux sont envisagées, le plus souvent de façon informelle. Des rapports de confiance les commandent.

Les coordinations hors territoire sont ponctuelles et les produits sont diffusés grâce aux contacts personnels de l'entrepreneur. Des relations de sous-traitance de compétence pour un donneur d'ordre régional peuvent cependant compléter l'activité : l'entreprise valorise ainsi son savoir-faire et augmente son chiffre d'affaires. L'entrepreneur reste cependant réticent à accroître ses effectifs pour répondre à une nouvelle demande.

Le comportement spatial est ici de proximité, alliant des relations personnelles territorialisées et parfois des relations industrielles hors territoire qui demeurent fragiles. L'ouverture à d'autres espaces est limitée. L'entreprise fait partie du territoire et se développe à proximité. Son insertion territoriale est forte.

\section{Des comportements délocalisés: les stratégies offensives et commandées}

Contrairement aux stratégies précédentes, l'espace des relations de l'entreprise engage avant tout des coordinations distantes. Deux pratiques sont concernées : les stratégies offensives pour lesquelles la croissance est prioritaire; les stratégies commandées par des sociétés dépendantes de $"$ groupes $^{9}$ ».

Les stratégies offensives concernent les tailles d'entreprises les plus importantes de l'échantillon (supérieures à 20 salariés, tableau 1). Elles sont d'origines diverses : nouvellement créées, développées sur plusieurs générations ou issues de délocalisations. Lorsque le créateur est ingénieur de formation, l'activité est lancée sur un savoir-faire et sur les réseaux tissés auprès des différentes communautés auxquelles il a appartenu ou appartient. Ce sont, dès le départ, des projets structurés qui réclament des investissements financiers importants. Leurs tailles demeurent cependant réduites pour les secteurs industriels touchés. L'entreprise recherche alors une différenciation sur le marché et des créneaux à investir, de techniques ou de

9. Ces établissements font ici partie de multinationales, de groupes, ou sont des entreprises au sein de holdings régionaux ou familiaux. 
Une SARL (secteur d'activité : industrie du cuir) de cinq personnes est créée à Paris en 1976 par un acteur local, après un apprentissage local et un compagnonnage. L'entreprise évolue et rapidement (10 ans plus tard) dissocie l'atelier de production des magasins pour réduire les coûts de main-d'œuvre ; en ville, les mesures sont faites sur l'handicapé, ainsi que l'essayage ; l'atelier de fabrication est implanté localement, dans le village natal de l'entrepreneur.

Actuellement, l'entreprise (SA) comprend une cinquantaine de salariés, une bonne implantation nationale, cinq magasins (dont deux à Paris) et une filiale (12 salariés). Le siège social se trouve localement sur le même site que l'atelier de production (30-32 personnes). Les produits et les prix sont fixés par un cahier des charges établi par la sécurité sociale.

L'entreprise se démarque des orthopédistes artisanaux classiques qui limitent leur activité à une clientèle de proximité. Elle développe un savoir-faire artisanal et une organisation de petite industrie, dissociant la production, la commercialisation et le contact avec la clientèle. L'activité se répartit entre des produits propres (s'adressant directement aux handicapés à travers les «boutiques »), des produits de série pour d'autres orthopédistes (pour la France et l'exportation, l'Allemagne et le Canada représentant $5 \%$ du chiffre d'affaires) et de sous-traitance pour trois ou quatre collègues français (10\% du chiffre d'affaires). L'entrepreneur envisage, enfin, de faire de son entreprise un centre de formation. Il espère ainsi favoriser une circulation d'informations plus intense, une meilleure connaissance d'éventuels clients et une opération promotionnelle pour l'entreprise. Le développement de ses activités est lié à la création prochaine d'une production de chaussures orthopédiques en kit, fournissant aux professionnels les pièces prédécoupées utilisables pour la réalisation de chaussures. Un débouché canadien est prévu.

savoir-faire particuliers (par exemple, technologies liées aux « hautes températures », édition d'ouvrages scientifiques), ou encore des brevets. L'image véhiculée par la production (réputation de l'entreprise) peut être un facteur de différenciation pour l'entreprise. Ces structures représentent plus de $30 \%$ de l'échantillon et se répartissent dans différents secteurs (tableau 2).

L'indépendance est une motivation forte chez l'entrepreneur et la croissance est souvent freinée par sa réticence à ouvrir le capital à d'autres actionnaires. Le dirigeant est dépositaire d'un patrimoine et les capitaux sont presque toujours familiaux. Cependant, au-delà du comportement patrimonial de certaines sociétés « dynastiques », la croissance demeure l'objectif premier. Le développement de l'entreprise se fait sur le long terme. Il y a accroissement de taille ou création d'unités indépendantes sur d'autres sites, témoignant de la dimension offensive de ces pratiques. Ainsi, dans le cas de certaines sous-traitance, l'entrepreneur envisage la croissance par la création de petits ateliers proches de donneurs d'ordres, pouvant bénéficier d'aides à la création. Dans tous les cas, les entreprises disposent d'un 
Cette entreprise (secteur d'activité : mécanique générale) a 15 salariés et fait partie d'un holding familial dont le centre de décision est à Paris. La création en 1980 de cette unité de sous-traitance est marquée par la volonté « politique » du p.d.-g., élu local, de créer une activité génératrice d'emplois locaux. Le projet est réalisé grâce à des primes de soutien à l'implantation.

Au départ, l'entreprise réalise uniquement de la sous-traitance : décolletage et mécanosoudure pour la société mère parisienne et divers travaux régionaux, représentant respectivement $40 \%, 40 \%$ et $20 \%$ du chiffre d'affaires 1982-1983. Les difficultés de la société parisienne et la réduction de sa part dans les activités de l'établissement - elle ne représente plus que $26,5 \%$ du chiffre d'affaires en 1990 - posent le problème du recentrage des activités. L'entreprise accroît alors le nombre de ses donneurs d'ordres, les diversifie et s'oriente vers des produits propres, produits brevetés rachetés par l'entreprise et mis en vente directe (ou sous d'autres marques).

L'entreprise s'adapte aux conditions nouvelles de son environnement économique par la modification de ses débouchés. La reconversion de l'entreprise aurait sans doute été plus brutale, si elle n'avait pas fait partie d'une structure plus importante. Actuellement, le chiffre d'affaires est constitué à $75 \%$ de la sous-traitance et à $25 \%$ des produits propres issus de rachats de brevets (un enrouleur de câble par achat de brevet, un trépied maçon).

L'entreprise demeure cependant essentiellement tournée vers le holding, où elle trouve son réseau de contacts ou de connaissances. Elle n'a pas développé d'autres circuits d'information ou de distribution de ses produits propres et doit résoudre des problèmes de commercialisation. L'importance du groupe reste primordiale dans la maîtrise de l'information, même si les salons professionnels et la presse spécialisée y concourent.

circuit commercial « classique » qui réunit des commerciaux salariés de l'entreprise et des agents multicartes. Elles créent des réseaux de distribution ou des sociétés commerciales indépendantes qui assurent la diffusion et le suivi de la production. La croissance liée au développement commercial dépend, enfin, de la capacité de l'entreprise à en assurer le financement (problème général aux petites structures). La vente de l'entreprise peut alors survenir pour conforter la croissance ou favoriser son développement.

Les stratégies commandées concernent les établissements secondaires créés par un groupe ou récemment rachetés (16\% de l'échantillon, tableau 1). Elles sont marquées, dans leurs orientations, par l'omniprésence d'un centre de décision délocalisé. Les entreprises rachetées, ayant développé un marché spécifique jugé intéressant, conservent leur propre clientèle, qui ne constitue guère plus de 5 à $10 \%$ de leurs débouchés. Les établissements ont pour clients essentiels d'autres établissements ou clients du groupe. Dans tous les cas, leur évolution dépend à terme des orientations du groupe. 
Les coordinations sont essentiellement marchandes et industrielles, même si les contacts personnels de l'entrepreneur jouent un rôle dans le développement et le maintien des marchés de l'entreprise. Ainsi, dans certains cas, elles font appel aux relations personnelles de l'entrepreneur, lesquelles lui permettent de bénéficier de circuits privilégiés sur un marché ou pour des approvisionnements. Ces relations sont alors de type coopératif et impliquent des acteurs ayant le plus souvent appartenu, comme le chef d'entreprise, à une même grande entreprise nationalisée, ou ayant eu la même formation. L'espace des relations ne répond plus à une continuité spatiale et la majorité des coordinations sont distantes. Ces relations délocalisées conduisent à une division spatiale des fonctions de production et de commercialisation. Ainsi, de nombreuses entreprises du secteur agroalimentaire ou parapharmaceutique commercialisent leur production grâce à un ensemble de points de vente en franchise ou appartenant à l'entreprise. Les relations de sous-traitance sont liées à la localisation du donneur d'ordres, rarement local, le plus souvent régional, parfois national. Face à la fragilité des coordinations industrielles entre sous-traitants et donneurs d'ordres, la proximité du donneur d'ordres conforte ces relations.

La maîtrise de l'information constitue un bon exemple des coordinations mises en place. Elle est liée à des coordinations distantes. Un premier accès à l'information est donné par les circuits commerciaux. Ceux-ci permettent des retours d'information sur la clientèle ou l'évolution de la demande, sur la concurrence et le marché. De nouvelles orientations pour la production sont alors possibles. Les représentants commerciaux et les VRP fournissent également à l'entrepreneur l'état du marché, permettant parfois l'acquisition de nouveaux marchés ou des retours d'appels d'offre. Un second accès à l'information est lié aux connaissances personnelles de l'entrepreneur. Dans un des cas rencontrés, des clients parisiens, venus au départ grâce à des connaissances personnelles des dirigeants ayant fait «boule de neige » (bouche à oreille) au niveau des ministères, constituent pour l'entrepreneur un marché captif. Pour les établissements, l'acquisition de l'information et de débouchés commerciaux est, dans tous les cas, centrée sur le groupe. Ce dernier constitue une source privilégiée d'échanges, formalisés ou non, d'informations concernant la technique, la recherche et développement. Au-delà du «filtre » que constitue le phénomène groupe, les pratiques s'apparentent alors à celles des entreprises offensives. D'une façon générale, l'accès à l'information passe par la construction sociale des relations et se développe dans le temps.

La main-d'œuvre qualifiée, flux important de l'entreprise, est recrutée par des circuits classiques (annonces, ANPE) ou des contacts professionnels régionaux, nationaux ou internationaux.

La proximité spatiale ne comporte donc que peu de coordinations. Celles-ci sont ténues et concernent l'accès aux ressources qui ont suscité l'implantation de l'entreprise. Ce sont des matières premières (extraction de carrières), une main- 
d'œuvre non qualifiée et des opportunités financières. Ces ressources ont une importance souvent ponctuelle dans le développement de l'entreprise. La relation au local peut également être fonctionnelle, fondée sur la proximité d'infrastructures de communication (autoroutes, aéroports) qui constituent un facteur recherché par l'entreprise, favorisant ses relations délocalisées. L'implantation est alors fragile et la présence locale de l'entreprise, incertaine. Pour les établissements, la question de la fragilité de l'implantation est amplifiée par la délocalisation du centre de décision. Ainsi, dans le cas de rachats d'entreprise par un groupe, celui-ci apporte dans un premier temps une logistique commerciale favorable à l'entreprise ; la perte d'indépendance peut conduire à une délocalisation de l'unité. L'histoire de l'entreprise et son développement local sont cependant des facteurs de pérennisation locale.

Ces comportements spatiaux, qu'il s'agisse des entreprises offensives (pour la plupart) ou des établissements secondaires, ne sont pas associés aux critères de proximité.

\section{Des comportements territorialisés: les stratégies adaptatives}

La majorité des entreprises concernées emploient moins de 20 salariés. Elles touchent de nombreux secteurs, en particulier l'agroalimentaire, l'industrie chimique et l'électronique, et concernent la majorité des entreprises étudiées (40\% de l'échantillon, tableau 1). Les entrepreneurs sont essentiellement des acteurs locaux et transposent un savoir-faire traditionnel (artisanal) à la petite industrie (petites séries). Les entreprises sont essentiellement patrimoniales. Il s'agit de petites entreprises capitalistes. Cependant, les investissements réguliers et conséquents requièrent des financements externes et une ouverture du capital. Certaines d'entre elles s'appuient largement sur le financement public lors de la réalisation du projet à travers les aides et les subventions. Pour ces entreprises se posent alors de nouveaux enjeux, en particulier celui du rendement de capital.

Le dirigeant a un souhait d'indépendance. La croissance de l'entreprise est limitée par sa volonté de demeurer sur un créneau, par une gestion «polyvalente» de l'entreprise ou encore par un choix de vie et de localisation. Il ne souhaite pas se transformer en «gestionnaire ». Il a souvent une démarche qui lie le développement industriel de son affaire à une implication personnelle au niveau du territoire : il est élu, membre d'une association... La volonté de pérennisation de l'entreprise est également présente même si elle n'apparaît pas primordiale. C'est le cas lorsque l'entrepreneur actuel appartient à une lignée familiale qui a développé la société sur plusieurs générations. Cependant, l'entreprise est souvent de création trop récente et le dirigeant trop jeune (entre 30 et 45 ans) pour penser à sa succession. 
Le chef d'entreprise recherche enfin la différenciation sur le marché de sa production : un créneau, une articulation de compétences spécialisées ou de savoirfaire, des partenariats. L'entreprise répond à une demande personnalisée, du « sur mesure ». Elle se spécialise dans des travaux complexes demandant une importante technicité. Elle investit des créneaux où le produit bénéficie d'une image « terroir », artisanale ou régionale. Ainsi, en agroalimentaire, un label ou une appellation d'origine lui permet de se positionner sur des marchés concurrentiels. Les secteurs en expansion, comme les sports «air et eau », offrent ponctuellement des situations analogues. Le créneau est alors trop spécifique pour que la concurrence soit forte, tant en taille d'entreprise ou en part de marché, qu'en technicité ou en qualité de produit. Selon les cas, les créneaux investis sont plus ou moins vulnérables.

Ces entreprises modulent leurs activités aux coopérations et aux réseaux qui leur assurent des débouchés privilégiés. Nous qualifions ces entreprises d'adaptatives.

\section{Réseaux et accès à l'information}

Les coordinations engagées sont personnelles, marchandes ou encore immatérielles. Le dirigeant fonde cependant l'essentiel de sa stratégie sur des coordinations personnelles et des coopérations.

L'accès à l'information sur les financements est encore une bonne illustration du phénomène. La maitrise qu'en a l'entreprise ne résulte pas tant d'une recherche systématique, mais davantage de mise en œuvre de contacts personnels de l'entrepreneur. Ainsi, la diversité des aides, des primes et des procédures d'obtention (multiplicité possible des interlocuteurs, manque de souplesse des critères d'accession) constituent un obstacle pour l'entreprise de petite taille qui n'a pas l'accès à l'information sur tous les financements possibles. Même si ses relations avec les financiers (banques, sociétés de capital-risque) sont essentiellement marchandes, les relations personnelles de l'entrepreneur, son intégration dans «les bons réseaux» et son implication dans les instances de décision sont alors déterminantes pour l'accès aux aides. Selon le projet et la capacité de l'entrepreneur à maîtriser les différentes sources d'information, les aides et les subventions peuvent représenter une large part du financement à la création. Différents niveaux de décision interviennent alors : communes, conseils général et régional, sociétés régionales de développement. Pour $90 \%$ des entreprises, les procédures les plus couramment utilisées sont celles du bâtiment-relais et de l'exonération de taxe professionnelle (trois à cinq ans).

Ainsi, de façon générale, l'accès à l'information est facilité par des contacts personnels au niveau d'institutions (Chambres de commerce et d'industrie, Chambres des métiers, organismes de développement, Conseil général). L'entrepreneur dispose également d'informations grâce aux «effets retour » de ses relations commerciales. L'information recherchée par l'entreprise concerne alors la demande (marchés et 
Cette SA au capital familial majoritaire (secteur d'activité : travail des métaux) de 12 salariés est reprise en 1989 par le fils. Au départ, seul le secteur agricole est envisagé. La baisse de cette activité conduit à élargir les débouchés. L'entreprise se spécialise alors dans la charpente métallique industrielle complexe ; elle est polyvalente et traite tout type de bâtiments complexes. L'entrepreneur dit cependant ne traiter que des « moutons à cinq pattes ». Cette compétence s'accompagne d'une souplesse de fonctionnement : partage de marchés avec d'autres entreprises, partenariats, réponses communes à des appels d'offre. Enfin, l'entreprise a une forte capacité d'adaptation à la demande. Son savoir-faire tient aux compétences présentes au sein de la structure et à la souplesse de fonctionnement.

La société s'est heurtée à la difficulté de se faire connaître et d'avoir accès à l'information. Peu de réponses aux appels d'offre locaux (dans un rayon de 60-70 km) sont données. En effet, les cahiers des charges ne mettent pas toujours en valeur le savoir-faire «travaux complexes » par lequel l'entreprise se différencie. D'autres formes d'accès aux marchés se sont alors développées. Des contacts professionnels et personnels forgés au cours des 20 ans d'activité du responsable lui permettent de valoriser son expérience. Ils se fondent sur la complémentarité des compétences et les relations de confiance. Ces réseaux constituent une ressource pour l'entreprise : il n'y a pas de prospection commerciale systématique, pas de plaquette publicitaire, peu d'appels d'offre traités directement. Ceux-ci sont en général abordés par l'intermédiaire de deux bureaux d'ingénierie sur Mende et Roanne, qui travaillent exclusivement avec l'entreprise lorsque des travaux de charpentes complexes sont demandés. Des partages de marchés sont également réalisés avec des artisans locaux. Enfin, des sous-traitances au niveau régional sont envisagées sur de gros marchés.

nouveaux produits), la technique (savoir-faire, recherche et développement) et les concurrents (parts de marchés détenues, gammes de produits présentées par d'autres entreprises).

\section{Articulations de coordinations territorialisées et coordinations délocalisées}

L'espace des relations de l'entreprise rassemble des coordinations territorialisées et délocalisées. Certaines relations personnelles de l'entrepreneur conduisent à des processus coopératifs et à la mise en commun de compétences et de ressources humaines. Leur qualité se construit alors dans le temps par la pratique et l'apprentissage ; elle résulte d'une construction collective, entre une demande et une production (une technique). Elle est enfin spécifique du processus engagé et ne pourrait être obtenue avec de quelconques partenaires. L'articulation entre coordinations territorialisées et délocalisées, selon les entreprises, s'effectue différemment. 
Les unes mettent en œuvre des coopérations locales et des réseaux de commercialisation extérieurs au territoire. Les coopérations impliquent des acteurs du territoire et l'appartenance à une même communauté culturelle et sociale.

C'est le cas des approvisionnements territoriaux de certaines entreprises agroalimentaires. L'entrepreneur est issu du territoire et de la profession agricole. Il mobilise autour de l'entreprise un ensemble d'agriculteurs. Les coordinations engagées s'appuient sur la reconnaissance de l'entrepreneur par les producteurs avec lesquels il est en relation contractuelle, sur son appartenance culturelle et sociale au territoire. La qualité des approvisionnements, leur régularité constituent l'une des clés de la stratégie de l'entreprise. Elle résulte de celle de la coordination. Le processus interactif mis en place se traduit par l'interdépendance de flux matériels et informels d'information. Il y a réciprocité de la relation entre le demandeur et les producteurs locaux ; les proximités sociales engagées sont territoriales. Les réseaux de commercialisations sont quant à eux régionaux ou nationaux. L'entreprise dispose de revendeurs ou de boutiques de vente pour la diffusion de produits se situant sur des créneaux (demandes ciblées, produit « artisanal » porteur de l'image du territoire). La relation entre l'entrepreneur et le vendeur se développe dans le temps.

Le deuxième type d'articulation des coordinations de l'entreprise met en œuvre l'insertion locale de l'entreprise (implication locale de l'entrepreneur, développement historique de l'entreprise, collaborations locales) et des coopérations (et réseaux) extérieures au territoire, fondées sur l'appartenance à des communautés professionnelles. Celles-ci s'appuient sur des collaborations passées de l'entrepreneur et de l'habitude de réalisations communes. Elles se concrétisent par des réponses communes à des appels d'offre grâce à une complémentarité de savoir-faire. La connaissance des compétences de chacun en assure une certaine pérennité.

Enfin, une troisième articulation des coordinations s'engage sur une synergie entre des coopérations territoriales et délocalisées. L'entreprise forge son savoirfaire sur des coordinations locales : elle adapte sa technique à une demande diversifiée et touche différents secteurs industriels locaux. Elle réalise des produits personnalisés et des prototypes en liaison avec la demande. L'originalité de son savoir-faire repose sur son adaptabilité et ses conceptions originales. L'entreprise le valorise en élargissant ses activités à des marchés extérieurs, relayés par des réseaux d'entreprises extérieures, connues grâce au parcours professionnel de l'entrepreneur.

Ces comportements spatiaux, au-delà de leurs particularités, articulent tous des coordinations territorialisées et délocalisées et s'appuient sur des proximités sociales. Nous parlerons d'ancrages territoriaux pour qualifier ces interactions. Ils sont de deux types. Les premiers concernent les relations personnelles que l'entrepreneur développe avec d'autres acteurs locaux. Certaines d'entre elles constituent des coopérations génératrices de ressources (compétences, mise en commun 
de ressources humaines) pour l'entreprise. Les seconds résultent de l'articulation entre des relations personnelles territoriales et des relations personnelles distantes. Elles ne peuvent être obtenues par simple contrat ou engagement écrit, mais font appel à des partenariats développés au cours du temps. Ceux-ci s'appuient sur des rapports de confiance, sur la réputation de chacun des protagonistes.

\section{Conclusion}

Nous avons montré la diversité des comportements spatiaux et leur lien avec la stratégie de l'entrepreneur, ses choix, son analyse de l'environnement, les coordinations qu'il y tisse.

Selon les stratégies, les modes d'insertion locale sont différents. Les entreprises artisanales présentent une immersion totale dans le territoire. Les entreprises adaptatives gèrent une articulation de relations locales et distantes. L'entreprise construit des liens sociaux avec des acteurs locaux et l'insertion au territoire est caractérisée par des échanges socialement construits, déterminants pour l'évolution de l'entreprise et pour la réduction de l'incertitude de la relation. Les entreprises offensives et les établissements de groupe évoluent vers des comportements délocalisés, où l'implantation engendre des relations essentiellement fonctionnelles, généralement en faible nombre, que l'entreprise pourrait retrouver ailleurs ou qui se révèlent ponctuelles pour elle. Les comportements s'appuient à terme sur des liens extérieurs; l'implantation est alors réduite à un espace de localisation sans enjeu capital pour l'entreprise. Les comportements spatiaux sont alors divers : localisés, ils limitent l'activité à une proximité spatiale; délocalisés, ils favorisent l'éclatement de l'espace des relations de l'entreprise; territorialisés, ils articulent des coordinations en s'appuyant sur des proximités sociales, territoriales et a-territoriales. Ils témoignent, tant par la nature des coordinations que par la localisation des acteurs impliqués, de la complexité des liens de l'entreprise à l'espace.

Une simple description de la localisation est alors loin de satisfaire la caractérisation de ces comportements. La diversité de ces derniers impose donc une analyse en termes d'interactions entre l'entreprise et les milieux ruraux. Certaines implantations demandent la proximité d'infrastructures de communication ; d'autres sont imbriquées dans des réseaux sociaux et sont envisageables en arrière-pays. Selon l'entreprise, tout territoire n'est pas approprié. Selon la localisation, tout projet n'est pas possible. La diversité des entreprises et des implantations le prouve. Les relations au local varient selon l'entreprise et ses attentes. Nous parlerons d'une nécessaire cohérence entre l'entreprise et le territoire, d'une adéquation entre le projet d'entreprise, l'entrepreneur (son comportement) et le territoire, système socioéconomique.

Enfin, ces résultats, obtenus selon un mode d'investigation adapté aux PE et aux TPE et à l'identification de l'entreprise à l'entrepreneur, demanderaient à être 
précisés pour des entreprises plus importantes. Au-delà de cette limite, ils introduisent cependant deux pistes d'investigation à valeur générique. La première est celle d'une construction par l'entreprise de son environnement.

L'entreprise sélectionne l'essentiel de ses relations. Les coordinations engagées ne sont pas de simples transactions liées à un coût, mais des relations dont l'organisation prend place dans la stratégie globale de la petite entreprise. Ainsi, les stratégies adaptatives témoignent de ce phénomène par les proximités sociales engagées. La construction de proche en proche de ces coordinations est alors envisagée à travers l' «imbrication» sociale marquant une distance avec une économie qualifiée de «sous-socialisée» (Granovetter, 1973, 1985) où les relations autres que fondées sur le seul intérêt marchand sont absentes. Elles font intervenir des formes coopératives, qui permettent à l'entreprise d'accéder à l'information sur le marché, la technique (recherche et développement) et les aspects financiers. Ces proximités sont territoriales donc d'abord culturelles, historiques et de traditions collectives. Elles peuvent aussi s'appuyer sur l'appartenance à des communautés professionnelles, à une même formation, à un parcours professionnel similaire ; elles impliquent alors des acteurs plus souvent éloignés. Ces proximités territoriales et a-territoriales permettent, par leur complémentarité, d'avancer l'idée de construction de l'environnement économique de l'entreprise.

La seconde piste de recherche porte sur le rôle des réseaux et des coopérations dans des processus de création de ressources de nature immatérielle notamment à travers des interactions sociales engagées. Un paramètre territorial est alors à considérer dans le positionnement sectoriel de l'entreprise.

\section{Bibliographie}

ARrow, K. J. (1974), The Limits of Organization, New York, Norton.

BAGNASCO, A. (1977), Tre Italie. La problematica territoriale dello sviluppo economico italiano, Bologna, Il Mulino.

BAUER, M. (1988), « Grands patrons, capital, État et entreprise : “ les 200 ” révèlent... », Sociologie du Travail, $\mathrm{n}^{\circ}$ 4, p. 567-585.

BeCATtini, G. (1989), «Les districts industriels en Italie», dans La flexibilité en Italie, Terr-Mire, p. 261-270.

BERTRAND, N. (1996), «Ancrage spatial des entreprises en milieu rural : de l'espace fonctionnel à l'espace territoire », Thèse de doctorat en économie appliquée, Université Pierre-Mendès-France Grenoble II, 12 juillet, 320 p.

BoltANSKI, L. et L. THÉVENOT (1991), De la justification. Les économies de la grandeur, Paris, Gallimard, 485 p.

COURLET, C. (1990), «Industrialisation et territoire, les systèmes productifs territorialisés », Colloque «Les districts industriels et la coopération interfirmes; enseignements et politiques à mettre en place», Trois-Rivières, GREPME, 3-5 avril, 36 p. 
COURLET, C. et B. PECQUeUR (1991), « Systèmes locaux d'entreprises et externalités : un essai de typologie », Revue d'Économie Régionale et Urbaine, $\mathrm{n}^{\mathrm{os}} 3 / 4$, p. 391-406.

DRUCKER, P. (1985), Les entrepreneurs, Paris, Hachette, Collection «Pluriel», 363 p.

GAROFOLI, G. (1992), «Les systèmes de petites entreprises : un cas paradigmatique de développement endogène », dans G. Benko et A. Lipietz (sous la direction de), Les régions qui gagnent, Districts et réseaux, les nouveaux paradigmes de la géographie économique, Paris, Presses universitaires de France, Collection «Économie en Liberté », $424 \mathrm{p}$.

GRANOVETTER, M.S. (1973), « The strength of weak ties », American Journal of Sociology, vol. $78, \mathrm{n}^{\circ} 6$, p. 1360-1380.

GRANOVETTER, M.S. (1985), «Economic action and social structure : the problem of embeddedness, American Journal of Sociology, vol. 91, nº 3, novembre, p. 481-510.

JAEGER, C. (1982), «Artisanat et capitalisme, l'envers de la roue de l'histoire», dans Artisanat et secteur des métiers, Paris, Payot, p. 176-198.

JOLY, P.B. et V. MANGEMATIN (1995), «Les acteurs sont-ils solubles dans les réseaux ?», Économies et Sociétés, Série Dynamique technologique et organisation, vol. 9, $\mathrm{n}^{\circ} 2$, p. 17-50.

Julien, P.A. et M. MARCheSnay (1987), La petite entreprise, Pars, Vuibert Gestion, 288 p.

KNIGHT, F.H. (1921), Risk, Uncertainty and Profit, New York, Houghton Mifflin Co.

LAWRENCE, P.R. et J.W. LORSCH (1973), Adapter les structures de l'entreprise, Paris, Éditions d'Organisation.

LECOQ, B. (1992), «Les districts industriels, une approche évolutionniste», Colloque international «Industrie et territoire : les systèmes productifs localisés », Grenoble, 21-22 octobre, $36 \mathrm{p}$.

MAILlAT, D. (1992), «Milieux et dynamique territoriale de l'innovation », Revue Canadienne des Sciences Régionales, vol. 15, $\mathrm{n}^{\circ} 2$, p. 199-218.

MARCheSnay, M. (1986), La stratégie, Paris, Chotard, 247 p.

MARCHESNAY, M. (1991), «Stratégie des petites et moyennes entreprises », Traité d'Économie Industrielle, $2^{\mathrm{e}}$ édition, Paris, Economica, p. 458-467.

Martinet, A.C. (1983), Stratégie, Paris, Vuibert, 322 p.

PERRIN, J.-C. (1992), «Pour une révision de la science régionale. L'approche par les milieux », Revue Canadienne des Sciences Régionales, vol. 15, n 2, p. 153-197.

PORTER, M.E. (1986), L'avantage concurrentiel, comment devancer ses concurrents et maintenir son avance, Paris, InterÉditions, $647 \mathrm{p}$.

RAFFESTIN, C. (1986), «Écogénèse territoriale et territorialité », dans F. Auriac et R. Brunet (sous la coordination de), Espaces, jeux et enjeux, Nouvelle encyclopédie des Sciences et des Techniques, Paris, Fayard, 343 p.

RALlET, A. et A. TORRE (1995), «Économie industrielle et économie spatiale : un état des lieux », dans Économie industrielle et Économie spatiale, Paris, Economica, 473 p.

RICHARDSON, G.B. (1972), « The organization of industry », The Economic Journal, vol. 82, $\mathrm{n}^{\circ} 327$, septembre, p. 883-896. 
SAlAIS, R. et M. TORPER (1993), «Les Mondes de Production, enquête sur l'identité économique de la France », Éditions de l'École des Hautes Études en sciences sociales, Paris, $467 \mathrm{p}$.

SAY, J.-B. (1803), Traité d'économie politique, Réédition en 1972, Paris, Calman-Lévy. SCHUMPETER, J. (1935), La théorie du développement économique, Traduction française, $4^{\mathrm{e}}$ édition de The Theory of Economic Development, Cambridge, University Press.

Simon, H.A. (1976), From Substantive to Procedural Rationality, Method and Appraisal in Economics, S. J. Latsis (éd.), réédition de Models of Bounded Rationality: Behavioral Economics and Business Organization, vol. 2, H.A. Simon, MIT Press, 1983, 505 p.

WeBer, H. (1988), «Cultures patronales et types d'entreprises : esquisse d'une typologie du patronat », Sociologie du travail, $\mathrm{n}^{\circ} 4$, p. 545-566. 